\title{
ESTUDIANTES MIGRANTES EN ESCUELAS PÚBLICAS CHILENAS ${ }^{1}$
}

\author{
Dante Castillo \\ Eduardo Santa-Cruz ${ }^{3}$ \\ Alejandro Vega ${ }^{4}$
}

RESUMEN

El artículo analiza el proceso de escolarización de los niños migrantes, sobre la base de las interrelaciones observadas entre los estudiantes, con el objeto de proporcionar insumos que fortalezcan la dinámica inclusiva del sistema escolar chileno. Su relevancia temática radica en los efectos en la integración social, consecuencia de la exclusión social de las minorías en el contexto escolar. Metodológicamente, es un trabajo descriptivocorrelacional, que explica las interrelaciones existentes entre los factores educativos y sociales, que permite comprender la dinámica escolar de los estudiantes migrantes y locales.

A través de un enfoque mixto, los resultados muestran la compleja red de representaciones sociales y escolares asociadas con la migración y la permanencia en el sistema escolar formal, así como las dificultades de inclusión de los colectivos migrantes en las dinámicas cotidianas de juego y trabajo en espacios escolares. Estas están relacionadas con la concentración de estudiantes migrantes en las aulas, su género y el colectivo específico al que pertenecen. El estudio también muestra que las instituciones escolares no tienen políticas específicas que organicen y estructuren lo que, muchas de ellas, intentan hacer en respuesta a la inclusión de la educación migratoria y a la interacción de colaboración entre los diferentes actores en el aula y las escuelas.

Conceptos clave: educación, inclusión, migración.

\section{IMMIGRANT STUDENTS IN CHILEAN PUBLIC SCHOOLS}

\section{ABSTRACT}

The article analyzes the school process of migrant children, based on observed interrelations between students, to provide insights that could strengthen inclusion dynamics within the Chilean school system. Its thematic relevance derives from the social consequences of the social exclusion of minorities in the school context. Methodologically, it is a descriptive-correlational work, which explains existing interrelations between educational and social factors, to understand the school dynamics of migrant and local students.

1 Este artículo es parte del proyecto "Escolarización de estudiantes migrantes en escuelas públicas chilenas", financiado por el Consejo Nacional de Educación - CNED.

2 Universidad Tecnológica Metropolitana. Santiago, Chile. Contacto: dante.castillo@utem.cl.

3 Universidad de Chile. Santiago, Chile. Contacto: eduardo.santacruz@ciae.uchile.cl.

4 Universidad Autónoma de Chile. Santiago, Chile. Contacto: alejandro.vega@uautonoma.cl 
Through a mixed approach, the results show the complex network of social and school representations associated with migration and retention in the formal school system, as well as the difficulties of including migrant collectives in the daily dynamics of play and work in school spaces. These difficulties are related to the migrant student's concentration in the classrooms, gender and the specific migrant collective to which he or she belongs. The study also shows that schools do not have specific policies that organize and structure what many try to do regarding the inclusion of immigrant students and collaborative interaction between the different actors in the classroom and schools.

Keyconcepts: education, inclusion, immigration.

\section{Antecedentes}

El actual ciclo migratorio chileno, iniciado a mediados de los años noventa, implicó que el país pasara de una sociedad emisora a otra receptora de migrantes, invirtiendo la tendencia de las décadas pasadas. La instalación democrática en Chile y el fin de la política de exilio, así como el crecimiento económico sostenido con tasas superiores al 5\%, generó las condiciones para que Chile se convirtiera en receptor de migrantes, provenientes principalmente de la región latinoamericana. La cifra de migrantes residiendo actualmente en el país, de acuerdo con los resultados del censo de población abreviado de 2017, se estimó en 750 mil personas, con un crecimiento significativo especialmente durante la última década. De hecho, solo el 6\% de estos llegó al país antes del año 1990, mientras que el 66\% lo hizo a partir de 2010. Este incremento en la población migrante se evidencia en las cifras: entre el censo de 1992 y el de 2002 su número creció un 77\%, mientras que entre los censos de 2002 y 2017, el incremento fue de casi un 300\% (Instituto Nacional de Estadísticas, INE, 2017).

De acuerdo con datos del censo abreviado de 2017, la población migrante latinoamericana es la más significativa. Existe una mayor presencia de personas provenientes de Perú $(25,3 \%)$; Colombia (14,2\%); Venezuela (11,2\%); Bolivia (9,9\%); Argentina $(8,9 \%)$ y Haití $(8,4 \%)$. Junto con ello, la Región Metropolitana es la que concentra la mayor cantidad de población extranjera, la que alcanza casi las 500 mil personas, cifra equivalente a un 7\% de su población. Sin embargo, en términos porcentuales su presencia es más relevante en las regiones de Tarapacá y Antofagasta, donde 
20 STUdiAnTES MigRANTES EN ESCUELAS PÚBLICAS CHILENAS - D. Castillo, E. SantaCruz y A. Vega

los migrantes corresponden al $14 \%$ y al $11 \%$ de la población, respectivamente. De acuerdo con datos anteriores, la mayor parte de la población migrante sería mayor a 15 años, mientras que una menor proporción estaría en edad escolar (INE, 2013).

Pese al crecimiento significativo y a la presencia que este fenómeno ha tenido en la agenda pública durante los últimos años, el país aún muestra un rezago importante en materia de políticas migratorias. Esta condición es observable en cada uno de los niveles del Estado (Stefoni y Fernández, 2011). El dato más relevante es que la normativa migratoria vigente data del año 1975 y el reglamento que la acompaña de 1984, esto es, respectivamente 20 y 10 años antes al inicio del actual ciclo migratorio que vive la sociedad chilena (Thayer, 2013).

Acerca de la implementación de políticas educativas orientadas a población migrante, el Ministerio de Educación de Chile, Mineduc, ha aprobado la Política Nacional de Estudiantes Extranjeros 20182022, donde se describe el conjunto de herramientas normativas e institucionales con que cuenta el sistema escolar para abordar el proceso de escolarización de los estudiantes migrantes. La construcción de esta política muestra el giro dado por el Estado central, ante la necesidad creciente de generar y coordinar acciones para atender la situación de esta nueva población presente en el sistema escolar. Sin embargo, hasta el momento, sus intervenciones han tenido un alcance bastante limitado, concentrándose principalmente en programas de regularización administrativa de niños y niñas migrantes matriculados en establecimientos públicos. La mayor parte de las iniciativas corresponden a acciones impulsadas por profesores, directores $u$ otros funcionarios de escuelas públicas, quienes han reaccionado de modo intuitivo a la transformación sociocultural que experimentan los establecimientos. También se registran acciones de carácter local, impulsadas de modo fragmentado por algunos sostenedores municipales donde la presencia migrante es más significativa.

Respecto de la magnitud del fenómeno migratorio en el sistema escolar, la evidencia indica que Chile aún se encuentra algo distante de lo ocurrido en otros contextos (Corporación Opción, 
2014), aun cuando en los últimos años las cifras han aumentado considerablemente. En ese sentido, solo entre los años 2015 y 2017 el número de estudiantes migrantes pasó de 30.625 a 77.608 en el conjunto del sistema escolar. A diferencia de la distribución de los estudiantes nacionales, donde ha crecido el número de estudiantes en el sector particular subvencionado, en el caso de los migrantes se ha ido produciendo una creciente concentración en establecimientos educativos de la red pública, pasando del 51\% en el año 2006 (Pávez, 2012) al 57\% durante el año 2016 (Mineduc, 2018). En cualquier caso, la tasa de estudiantes migrantes en la red pública se encuentra actualmente en un 3,5\%, cifra menor comparada con la experiencia de países que se han caracterizado por ser sociedades receptoras. Ahora bien, al interior de la propia red pública también se dan procesos de concentración de magnitud. Por ejemplo, en la comuna de Quilicura, dos de los 14 establecimientos públicos poseen el 44\% de los estudiantes inmigrantes (Thayer, 2013). En esta misma comuna, otro aspecto relevante es la existencia de un elevado número de estudiantes en condición irregular (57\%), mientras que otro tanto (20\%) no cuenta con registro de nacionalidad. Este fenómeno también se aprecia a nivel nacional, donde un tercio de los estudiantes se encontraría en condición migratoria irregular y no contaría con visa nacional o registro de nacionalidad (Mineduc, 2018).

Pese al creciente conocimiento acerca de la distribución de los estudiantes migrantes existe poca evidencia de las características, perfiles y forma en la cual viven sus procesos de escolarización. Por este motivo, comprender los procesos migratorios en los que niños, niñas y adolescentes se ven involucrados resulta esencial y, además, un aspecto complejo de estudiar.

\section{Aspectos conceptuales}

La investigación tiene por objeto comprender cómo afecta la condición social de migrante en el proceso de incorporación al sistema escolar, específicamente, en la escolarización de niños y niñas en el segundo ciclo básico en escuelas de comunas con alta presencia de población migrante. El alcance de esta interrogante está delimitado en supuestos teóricos que se fundamentan a continuación. 
2.1. La inclusión de migrantes: los modos de incorporación El campo de los estudios migratorios en la actualidad, tanto en Latinoamérica como Europa y EE.UU., está marcado por la tendencia, crecientemente hegemónica, de poner en un papel cada vez más protagónico a los vínculos y prácticas transnacionales de los migrantes, y matizar o dar por superada en algunos casos, la idea tradicional de que la migración supone que los sujetos salen de un país de origen para radicarse definitivamente en el de destino. Desde este enfoque transnacional, la migración se entiende como una dinámica en la que el migrante forma parte de un espacio que involucra simultáneamente los contextos de procedencia y recepción (Castles \& Davidson, 2000).

El estudio del proceso de inclusión de los migrantes en las sociedades de destino, desde el enfoque de los modos de incorporación (Portes y Rumabaut, 1990), surge como respuesta a dos insuficiencias en las perspectivas analíticas disponibles, derivadas a su vez de las teorías de la asimilación desarrolladas a partir del trabajo fundante de la Escuela de Chicago a principios del siglo XX y del enfoque neoclásico (Borjas, 1995). En primer lugar, la evidencia empírica reunida en diversas investigaciones demostró que la hipótesis elaborada por el paradigma clásico de la asimilación, que asume el proceso de aculturación migrante como garantía de acceso igualitario a oportunidades, derechos y recursos disponibles para la movilidad social de migrantes y sus hijos (Park y Burguess, 1969), no era aplicable a la realidad de los flujos migratorios contemporáneos (Portes, 1990). En segundo lugar, las investigaciones mostraron la poca validez de la tesis neoclásica que planteaba que la movilidad ascendente de los migrantes y su desempeño económico depende exclusivamente de los niveles de educación y experiencia laboral (Portes, 1995).

En contra de estas tesis clásicas, el trabajo realizado por Portes acerca de una serie de colectivos de migrantes en la sociedad norteamericana mostró que los procesos de asimilación en lugar de estar conduciendo a los migrantes a un acceso igualitario al mercado laboral, los estaba llevando en la dirección contraria. No solo la asimilación había dejado de ser un instrumento eficiente para la 
movilidad socioocupacional ascendente, sino además quedaba cada vez más en evidencia que:

...los jóvenes inmigrantes que permanecían fuertemente integrados en sus respectivas comunidades étnicas podían tener, en virtud de este hecho, mayores oportunidades de movilidad educacional y social, a través del uso del capital material y social que sus comunidades hacían posible (Portes y Zhou, 1993, pp. 81-82).

Este hallazgo es concordante con los resultados que, desde una mirada económica, Stark (1993) presentó a partir de un análisis del acceso a recursos en colectivos de calificación baja y media en la sociedad norteamericana.

Frente a esta evidencia, Portes y Zhou (1993) plantean la existencia de una "incorporación segmentada", que dependería de los modos de incorporación de los distintos colectivos de migrantes. Los autores reconocen tres modos paradigmáticos posibles en las sociedades receptoras: el primero, combina la adaptación cultural de los migrantes con un acceso creciente a los recursos análogos de las clases medias locales. El segundo, consiste en una incorporación de los migrantes a condiciones de pobreza extrema, lo que supone la entrada y posterior permanencia en las posiciones inferiores de la escala sociolaboral. El tercer modo consiste en el acceso al bienestar económico sobre la base de la preservación deliberada de los lazos, los valores y la solidaridad al interior de su comunidad, lo que se podría definir como acceso a los recursos sin adaptación cultural.

A partir de esta tipología surge la pregunta en torno a qué factores inciden en que los migrantes sigan alguno de estos tres caminos en su entrada a la sociedad receptora (Portes y Zhou, 1993). La propuesta analítica aborda esta pregunta centrándose en las condiciones sociales, políticas y culturales en las que están insertos los inmigrantes, y cómo esta inserción afecta positiva o negativamente sus posibilidades de acceder en igualdad de condiciones al bienestar social. En tal sentido, cabe recordar que: 
Los inmigrantes no son vistos simplemente como individuos que poseen ciertas habilidades personales, sino como miembros de grupos y partícipes en estructuras sociales mayores que afectan en múltiples sentidos su movilidad económica. El concepto de modos de incorporación se refiere al proceso de inserción de los inmigrantes en estos variados contextos sociales. Los efectos contextuales interactúan con el capital traído desde el extranjero, determinando el alcance en que puede ser productivamente utilizado o incrementado (Portes, 1995, p. 24).

La propuesta analítica consiste en un modelo basado en tres variables que configuran el contexto de inserción de los migrantes, las que inciden en el destino de su trayectoria: la primera es la política gubernamental del Estado receptor, que podría oscilar entre "receptiva, indiferente y hostil". Esta variable da cuenta del hecho que el Estado no enfrenta de igual manera a los distintos colectivos migratorios, y que es necesario considerar su accionar, en el plano de las políticas de control fronterizo y las políticas sociales de acogida y reconocimiento de derechos.

La segunda variable dice relación con la disposición de la sociedad local y los actores organizados para la receptividad de los distintos colectivos. La literatura distingue dos categorías en relación con esta disposición receptiva: prejuiciosa/discriminatoria y desprejuiciada/neutral. El supuesto es que los diversos colectivos son recibidos de distinto modo según: origen nacional, condición cultural, rasgos físicos, etc.

La tercera variable que definiría el contexto de inserción es la conformación social del colectivo nacional de inserción del migrante, se distinguen al respecto comunidades fuertes o débiles. Lo que se define por sustrato de clase, el nivel medio de instrucción y la magnitud cuantitativa del colectivo. Son consideradas débiles las comunidades pequeñas que están conformadas principalmente por trabajadores manuales y de baja calificación, y fuertes aquellas numerosas y diversificadas en cuanto al carácter socioocupacional de sus componentes, que tienen en general, un promedio de instrucción medio o alto (Portes y Zhou, 1993). 


\subsection{Procesos de escolarización de los inmigrantes}

La evidencia respecto de los procesos de escolarización de la población migrante cuenta con un mayor desarrollo en países donde los procesos migratorios tienen más larga data. En el caso de Chile, la investigación es aún insuficiente, pese a que mucha de ella tiende a estar alineada con la evidencia internacional.

En función de los objetivos de esta investigación, se presentan algunos de los hallazgos encontrados, tanto en Chile como en otros contextos, en tres dimensiones relevantes de la experiencia escolar:

- cultura escolar, en particular en relación con la existencia de dinámicas de discriminación al interior de los establecimientos escolares y dinámicas institucionales tendientes a enfrentarlas;

- rendimiento académico de la población migrante; y

- distribución y grado de concentración territorial y en la red educativa de la población migrante y de los nacionales.

Uno de los tópicos recurrentes en el debate acerca de inmigración y escuela es la tensión existente entre la consolidación de prejuicios y estereotipos y la capacidad de la institución escolar para morigerarlos. Cárdenas (2006) detectó en una muestra de enseñanza media que los prejuicios abiertamente hostiles habían derivado en otros más sutiles o encubiertos, pero no habían desaparecido, corroborando la hipótesis de Pettigrew y Meertens (1995) de que existe dificultad en reconocerse a sí mismos como racistas o xenófobos. En otras palabras, la mera convivencia no elimina la existencia de discriminación en la cotidianidad escolar. Para España, Thayer (2007) detectó que la estigmatización al interior de los espacios escolares es menor si la cantidad de inmigrantes es mayor, dada la percepción entre los nacionales de la capacidad de los migrantes para disputarles el control sobre el espacio escolar. Este prejuicio sutil también se detectó en estudiantes de Pedagogía en Chile, con menor intensidad en mujeres y personas con interacciones previas con inmigrantes (Navas y Sánchez, 2010). Esta discriminación se evidencia también en formas menos sutiles, que se despliegan en lo cotidiano en el habla de los adultos hacia los niños, niñas y adolescentes, categorizándolos y clasificándolos (Tijoux, 2013). Pese 
a esto, como sostiene la autora, la llegada de la inmigración cumple una función económica, lo mismo que en muchas ciudades europeas (Alegre, Benito \& González, 2006; Burgess, Wilson \& Lupton, 2005), donde el ingreso masivo de estos estudiantes ayuda a sobrevivir al agónico sistema público en aquellos barrios donde las familias más acomodadas han decidido enviar a sus hijos a colegios particulares subvencionados. En el caso chileno se expresa en que la llegada de estudiantes migrantes a la red pública ha permitido invertir la tendencia de pérdida de matrícula de casi dos décadas.

Un segundo aspecto relevante se relaciona con el efecto de la inmigración en los resultados académicos. De acuerdo con Calero y Waisgrais (2009), solo se detectan efectos negativos en concentraciones muy elevadas de inmigrantes. Abrantes (2008), también para el caso español, da cuenta de que los y las inmigrantes tienen mayor dificultad para adaptarse a contextos más académicos y selectivos, donde las clases expositivas y el estudio en casa se vuelven hegemónicos. Rivkin (2000) proporciona evidencia de que el aislamiento racial perjudica el logro académico. La mayoría de los países miembros de la Organización para la Cooperación y el Desarrollo Económico, OCDE, los y las estudiantes inmigrantes registran un rendimiento inferior respecto del resto de sus compañeros. No obstante, diferentes experiencias internacionales han demostrado que dicha brecha educacional se ha podido reducir considerablemente, como en el caso de Bélgica, Suiza, Alemania y Nueva Zelanda; e incluso revertir, como en el de Australia (OCDE, 2011). De este modo, las bajas expectativas y los estereotipos de la comunidad educativa, así como las políticas de los centros educacionales, explicarían de mejor modo el bajo rendimiento de los y las menores inmigrantes. En este plano, cabe indicar que no existen culturas deficientes, sino incompatibilidades entre la cultura familiar del estudiante y las expectativas de la escuela (Gallimore \& Goldenberg, 2001). Tal como sostuvo Bernstein (1994), puede ser que determinadas configuraciones escolares se adapten mejor a ciertos contextos familiares.

En relación con el último ámbito, la evidencia comparada muestra que existe una recurrente concentración y segregación de 
la población migrante, en particular, de aquellos que carecen de capital simbólico, pero también de los que poseen mayor capital económico y se autosegregan (Schiappacasse, 2008). Esta misma tendencia también la observaban Frankenberg, Lee y Orfield (2003) en Estados Unidos, aun cuando este problema no se presenta de un modo homogéneo para todos los colectivos, siendo los latinos el grupo más segregado y los asiáticos el grupo menos segregado.

Por último, el estudio del sistema educativo se ha centrado en la relación entre cultura y cohesión social, y en las condiciones de desigualdad y de mayor precariedad socioeconómica que afectan a la población migrante. Los trabajos situados en esta línea han sido enfáticos en precisar la necesidad de considerar, simultáneamente, la cuestión de la cohesión social a partir de la existencia de diferencias culturales y el problema de la desigualdad social que instala a los migrantes en una posición subordinada respecto de la población local (Abad, 1993a; García y Granados, 2002; Colectivo Ioé, 2002). Los especialistas plantean que, de no considerar simultáneamente estos dos aspectos, el sistema educativo contribuiría a reproducir esas condiciones de partida, en lugar de revertirlas.

En el ámbito de los modelos de intervención escolar orientados a generar un marco de inclusión social de los migrantes, es posible distinguir cinco estrategias de intervención por parte de las instituciones educativas (Colectivo Ioé, 1999a, 1999b; Giménez y Malgesini, 2000).

- En la primera, la escuela activa un rechazo a los diferentes negando el acceso a los migrantes, evitando hacerse cargo de su escolarización y su socialización.

- Una segunda posición supone ignorar a los diferentes, permitiendo el ingreso de los migrantes a la escuela, tratándolos como si no fueran portadores de una diferencia cultural, ni estuvieran en una posición de desigualdad social.

- En tercer lugar, la escuela los puede educar mediante programas compensatorios y planes especiales, nivelando la situación de partida de aquellos que, por su condición social y cultural, se encuentran en una posición desfavorecida. 
- En cuarto lugar, la escuela puede potenciar una educación pluralista, introduciendo en el currículo las aportaciones culturales de los distintos colectivos de migrantes.

- Por último, se ha identificado el modelo de la educación antirracista o intercultural, que toma los planteamientos de la educación pluralista y los asocia a la posición de poder desfavorable en que se encuentran las minorías migrantes.

Esta última estrategia estaría orientada tanto a la inclusión de las singularidades culturales, como a suprimir las desigualdades sociales y de poder que afectan negativamente a migrantes y a otras minorías (Colectivo Ioé, 1999a; Giménez y Malgesini, 2000).

\section{Metodología}

La investigación que da origen a este artículo incorporó un enfoque mixto, considerando el uso de técnicas cuantitativas y cualitativas. La unidad de análisis correspondió a estudiantes y apoderados migrantes y no migrantes de escuelas públicas de la Región Metropolitana. La muestra cuantitativa utilizó un procedimiento de selección probabilístico, basado en un muestreo por conglomerados con un procedimiento de selección polietápico. El marco muestral se construyó a partir del procesamiento de datos estadísticos nacionales, permitiendo identificar cuatro conglomerados geográficos, correspondientes a cuatro municipios de Santiago de Chile con alta población escolar migrante. Tal como se indica en la Tabla 1, al interior de dichas comunas se seleccionaron a su vez a diez escuelas con diferentes grados de concentración de estudiantes migrantes, de primera y segunda generación. 
Tabla 1

Distribución de la matrícula de segundo ciclo básico según grupos de interés

\begin{tabular}{|c|c|c|c|c|c|c|c|}
\hline \multirow{3}{*}{ Comunas } & \multirow{3}{*}{$\begin{array}{c}\text { Nombre del } \\
\text { establecimiento }\end{array}$} & \multirow{3}{*}{ Dependencia } & \multicolumn{5}{|c|}{$\begin{array}{l}\text { Matrícula solo } 2^{\text {do }} \text { ciclo } \\
\qquad\left(5^{\circ}-8^{\circ}\right)\end{array}$} \\
\hline & & & \multicolumn{2}{|c|}{$\begin{array}{c}\text { Estudiantes } \\
\text { migrantes }\end{array}$} & \multicolumn{2}{|c|}{$\begin{array}{l}\text { Estudiantes } \\
\text { nacionales }\end{array}$} & \multirow{2}{*}{$\begin{array}{c}\begin{array}{c}\text { Total } \\
\text { matrícula } \\
2^{\text {do }} \text { Ciclo }\end{array} \\
N^{\circ}\end{array}$} \\
\hline & & & $\mathrm{N}^{\circ}$ & $\%$ & $\mathrm{~N}^{\circ}$ & $\%$ & \\
\hline Independencia & Escuela 1 & Municipal & 68 & $50 \%$ & 68 & $50 \%$ & 136 \\
\hline Quilicura & Escuela 2 & Municipal & 82 & $34 \%$ & 160 & $66 \%$ & 242 \\
\hline Santiago & Escuela 3 & Municipal & 76 & $30 \%$ & 177 & $70 \%$ & 253 \\
\hline Recoleta & Escuela 4 & Municipal & 172 & $29 \%$ & 424 & $71 \%$ & 596 \\
\hline Independencia & Escuela 5 & Municipal & 66 & $19 \%$ & 275 & $81 \%$ & 341 \\
\hline Estación Central & Escuela 6 & Municipal & 20 & $17 \%$ & 97 & $83 \%$ & 117 \\
\hline Estación Central & Escuela 7 & Municipal & 18 & $14 \%$ & 115 & $86 \%$ & 133 \\
\hline Santiago & Escuela 8 & Municipal & 34 & $11 \%$ & 287 & $89 \%$ & 321 \\
\hline Santiago & Escuela 9 & Municipal & 22 & $8 \%$ & 259 & $92 \%$ & 281 \\
\hline \multirow[t]{2}{*}{ Recoleta } & Escuela 10 & Municipal & 9 & $3 \%$ & 260 & $97 \%$ & 269 \\
\hline & & Total: & 567 & & 2.122 & & 2.689 \\
\hline
\end{tabular}

Fuente: Elaboración propia.

A continuación, se administró una encuesta a los apoderados, considerando un error máximo admisible de 5\% y un 95\% de confianza. Para este instrumento, el marco muestral estuvo compuesto por 2.689 apoderados migrantes y no migrantes. La muestra estratificada fue administrada a 534 apoderados migrantes y no migrantes. Para el test sociométrico, se seleccionaron aleatoriamente 20 cursos de $5^{\circ}$ a $8^{\circ}$ año básico, dos cursos por escuela, aplicándose a una muestra de 663 estudiantes. Para proteger la integridad de los datos y su procesamiento se hizo modificando la identidad de los estudiantes, previa solicitud a sus familias de la autorización para la recogida y el uso de datos por parte del equipo investigador. Para el procesamiento de la información se siguieron dos caminos: elaboración de tipos sociométricos y construcción de sociogramas. En relación con lo primero, y siguiendo a García-Bacete, Sureda y Monjas (2010), se construyeron tipologías sociométricas, mediante el procesamiento de los indicadores individuales sociométricos, en concreto las elecciones y rechazos recibidos por cada individuo, dando lugar a cinco tipos de sujetos. Estos son: 
30 STUdiAnTES MigRANTES EN ESCUELAS PÚBLICAS CHILENAS - D. Castillo, E. SantaCruz y A. Vega

1. Preferidos, que corresponden a los individuos que reciben elecciones positivas significativamente altas y bajos o nulos rechazos.

2. Rechazados, son quienes reciben nominaciones negativas significativamente altas y un número bajo de nominaciones positivas.

3. Ignorados, reciben escasas o ninguna nominación positiva o negativa por parte de sus compañeros.

4. Controvertidos, son quienes reciben nominaciones positivas y negativas en un número elevado, superior a la media de elecciones y rechazos.

5. Promedio, es el tipo de sujeto que no cumple ninguna de las anteriores condiciones.

También se elaboró un sociograma por grupo-curso (Bezanilla, 2011), con el objeto de graficar las distintas estructuras y dinámicas de relaciones intergrupales e intragrupales dentro de un mismo curso.

\section{Resultados}

\subsection{Perfiles familiares y contextos}

Los perfiles de las familias migrantes y nacionales muestran similitudes y diferencias que son relevantes para comprender su acercamiento a la experiencia escolar de sus hijos. Como se ha señalado con anterioridad (Castillo y Gajardo, 2016), se conoce poco acerca de las características de dichas familias y de sus expectativas en torno a los procesos de escolarización, componente que es fundamental para comprender el modo en que ellas se incorporan a la sociedad de acogida. A partir de la encuesta aplicada a apoderados migrantes y no migrantes, en este apartado se presentan algunos datos sobre rasgos personales, condición socioeconómica y estatus laboral, capital cultural objetivado e institucionalizado, trayectoria de los estudiantes migrantes, expectativas familiares del futuro escolar de sus hijos y percepción de discriminación social hacia los migrantes.

Un rasgo tradicional en el sistema escolar chileno es que el rol de representación frente a la escuela lo suele asumir la madre, como parte de la distribución histórica de los trabajos domésticos y la función de cuidado de los hijos. En el caso de las familias migrantes, 
esta desigual distribución respecto del rol de apoderado se mantiene, aun cuando la proporción de hombres que asumen este trabajo es significativamente mayor que en el caso de las familias nacionales (24,6\% en migrantes frente a $12,3 \%$ en nacionales). Esta diferencia se puede explicar, debido a la mayor participación en el mercado de trabajo de las mujeres migrantes, lo que les impide poder asumir dicho rol dadas las largas jornadas laborales.

Esta primera diferencia en las familias se complementa con la desigual distribución en grupos etarios de ambos colectivos. Tal como aparece en la Tabla 2, los apoderados migrantes se concentran preferentemente entre los 30 y 40 años $(63,9 \%)$, mientras que los apoderados nacionales tienden a mostrar una mayor edad promedio, con alta presencia entre los 41 y los 60 años.

Tabla 2

Grupos etarios y nacionalidad

\begin{tabular}{lccc}
\hline Grupos etarios & \multicolumn{2}{c}{ ¿Es usted extranjero? } & Total \\
\hline & Sí & No & \\
Hasta 24 años & 1,6 & 1,2 & 1,3 \\
Entre 25 y 29 años & 3,2 & 8,0 & 6,4 \\
Entre 30 y 40 años & 63,9 & 46,9 & 52,5 \\
Entre 41 y 60 años & 30,2 & 43,2 & 38,9 \\
Más de 60 años & 1,2 & 0,8 & 0,9 \\
Total & 100,0 & 100,0 & 100,0 \\
\hline
\end{tabular}

Fuente: Elaboración propia.

Respecto de la condición socioeconómica, los datos muestran que los apoderados migrantes suelen realizar trabajos de baja cualificación, aun cuando no existen diferencias significativas, como se verá más adelante, en el nivel de instrucción formal de ambos colectivos. En el caso de los hombres migrantes, existe una elevada concentración en el sector servicios (30\%) o en la categoría de ocupación no calificada (18\%), mientras que en el caso de las mujeres migrantes es el servicio doméstico (34\%) y dueña de casa (22\%), las que evidencias mayor concentración. Como se observa en la Tabla 3, esto marca una importante diferencia con los apoderados nacionales, quienes han podido escapar progresivamente de las labores de menor calificación. Este acceso subordinado al mercado 
32 STUdiantes MigRANTES EN ESCUELAS PÚBLiCAS CHILENAS - D. Castillo, E. SantaCruz y A. Vega

de trabajo por parte del colectivo migrante no se puede atribuir a un menor capital cultural, pues no existen diferencias sustantivas respecto de los nacionales.

Tabla 3

Ocupación principal de padres y madres de estudiantes

\begin{tabular}{|c|c|c|c|c|c|c|}
\hline \multirow{3}{*}{$\begin{array}{l}\text { Ocupación principal; padre (padrastro) } \\
\text { del estudiante }\end{array}$} & \multicolumn{3}{|c|}{ Padre } & \multicolumn{3}{|c|}{ Madre } \\
\hline & \multicolumn{2}{|c|}{$\begin{array}{l}\text { ¿Es usted } \\
\text { extranjero? }\end{array}$} & \multirow[t]{2}{*}{ Total } & \multicolumn{2}{|c|}{$\begin{array}{l}\text { ¿Es usted } \\
\text { extranjero? }\end{array}$} & \multirow[t]{2}{*}{ Total } \\
\hline & Sí & No & & Sí & No & \\
\hline $\begin{array}{l}\text { Legislador, funcionario de alto nivel de } \\
\text { gobierno y/o administración pública. }\end{array}$ & 1,1 & 1,9 & 1,6 & - & 0,6 & 0,4 \\
\hline $\begin{array}{l}\text { Miembro de las Fuerzas Armadas y del } \\
\text { Orden. }\end{array}$ & - & 1,2 & 0,8 & - & 0,6 & 0,4 \\
\hline $\begin{array}{l}\text { Empresario, directivo y/o alto ejecutivo de } \\
\text { empresa. }\end{array}$ & 1,1 & 1,4 & 1,3 & - & 0,6 & 0,4 \\
\hline Personal administrativo de oficina. & 3,2 & 13,5 & 10,4 & 2,7 & 14,0 & 10,4 \\
\hline Profesional universitario. & 0,5 & 8,6 & 6,2 & 1,4 & 6,4 & 4,8 \\
\hline Técnico o profesional de nivel técnico. & 6,9 & 14,7 & 12,3 & 1,4 & 10,2 & 7,4 \\
\hline Trabajador de servicios y/o comercio. & 30,3 & 17,5 & 21,4 & 10,4 & 16,5 & 14,6 \\
\hline Operador de maquinaria o ensamblador. & 6,4 & 6,8 & 6,6 & 2,7 & 0,8 & 1,4 \\
\hline $\begin{array}{l}\text { Trabajador calificado del sector agrícola o } \\
\text { la pesca. }\end{array}$ & 0,5 & 0,2 & 0,3 & 0,0 & 0,0 & 0,0 \\
\hline Artesano. & 0,5 & 0,7 & 0,6 & 1,8 & 0,6 & 1,0 \\
\hline $\begin{array}{l}\text { Servicio doméstico de puertas adentro o } \\
\text { afuera. }\end{array}$ & 2,1 & 0,2 & 0,8 & 34,4 & 3,8 & 13,6 \\
\hline Ocupación no calificada. & 18,6 & 8,9 & 11,8 & 8,1 & 3,6 & 5,1 \\
\hline Dueño/a de casa. & - & 0,9 & 0,6 & 22,2 & 27,1 & 25,5 \\
\hline Familiar no remunerado. & 0,5 & 0,2 & 0,3 & 0,9 & 0,4 & 0,6 \\
\hline Estudiante. & - & 0,2 & 0,2 & 0,5 & 1,7 & 1,3 \\
\hline Sin ocupación. & 3,7 & 2,8 & 3,1 & 4,1 & 3,6 & 3,8 \\
\hline Otra. & 24,5 & 20,0 & 21,4 & 9,5 & 9,1 & 9,2 \\
\hline No contesta. & - & 0,2 & 0,2 & - & 0,2 & 0,1 \\
\hline Total & 100,0 & 100,0 & 100,0 & 100,0 & 100,0 & 100,0 \\
\hline
\end{tabular}

Fuente: Elaboración propia.

Vinculado con lo anterior, los ingresos familiares expresados por los apoderados migrantes son inferiores a los de sus pares nacionales. Por ejemplo, mientras un 10,2\% de los apoderados nacionales declara obtener entre 800 mil y 1 millón doscientos mil pesos de ingreso familiar, esta cifra se reduce al $2 \%$ en el caso de las familias migrantes. De hecho, $68,7 \%$ de las familias migrantes declaran tener menos de 400 mil pesos como núcleo familiar, en contraposición con el $57 \%$ que registran las familias nacionales. 
Tabla 4

Ingresos del hogar del estudiante

\begin{tabular}{lrrr}
\hline \multirow{2}{*}{ Ingreso del hogar } & \multicolumn{2}{c}{ ¿Es usted extranjero? } & \multirow{2}{*}{ Total } \\
\cline { 2 - 3 } & \multicolumn{1}{c}{ Sín } & \multicolumn{1}{c}{ No } & \\
\hline Menos de $\$ 200.000$ & 14,1 & 10,8 & 11,9 \\
Entre $\$ 200.001$ y $\$ 400.000$ & 54,6 & 46,6 & 49,2 \\
Entre $\$ 400.001$ y $\$ 800.000$ & 28,3 & 27,6 & 27,8 \\
Entre $\$ 800.001$ y $\$ 1.200 .000$ & 2,0 & 10,2 & 7,5 \\
Entre $\$ 1.200 .001$ y $\$ 1.800 .000$ & 0,4 & 3,0 & 2,1 \\
Sobre $\$ 1.800 .001$ & 0,4 & 1,8 & 1,3 \\
Total & 100,0 & 100,0 & 100,0 \\
\hline
\end{tabular}

Fuente: Elaboración propia.

En relación con el capital cultural, observamos que las credenciales educativas obtenidas por los migrantes presentaron diferencias significativas con la distribución que se observa entre los nativos, entre apoderados de ambos sexos. Un aspecto importante de relevar es la alta proporción, en ambas poblaciones, de apoderados que declaran haber cursado estudios de educación superior. Ahora bien, por limitaciones del instrumento, no existe certeza si estas experiencias se completaron y de qué nivel son.

Tabla 5

Nivel educacional de los apoderados

\begin{tabular}{|c|c|c|c|c|c|c|}
\hline \multirow{3}{*}{ Nivel educativo } & \multicolumn{3}{|c|}{ Padre } & \multicolumn{3}{|c|}{ Madre } \\
\hline & \multicolumn{2}{|c|}{$\begin{array}{l}\text { ¿Es usted } \\
\text { extranjero? }\end{array}$} & \multirow[t]{2}{*}{ Total } & \multicolumn{2}{|c|}{$\begin{array}{l}\text { ¿Es usted } \\
\text { extranjero? }\end{array}$} & \multirow[t]{2}{*}{ Total } \\
\hline & Sí & No & & Sí & No & \\
\hline Educación básica & 14,4 & 12,6 & 13,1 & 16,5 & 13,9 & 14,7 \\
\hline Educación media & 47,6 & 50,4 & 49,5 & 48,1 & 48,4 & 48,3 \\
\hline Educación superior & 38,0 & 37,0 & 37,3 & 35,4 & 37,7 & 36,9 \\
\hline Total & 100,0 & 100,0 & 100,0 & 100,0 & 100,0 & 100,0 \\
\hline
\end{tabular}

Fuente: Elaboración propia.

En relación con el capital cultural incorporado, el cual se puede expresar en la disponibilidad de libros en el hogar de los estudiantes, se observan diferencias significativas entre las familias migrantes y nacionales. Considerando la similitud de trayectorias educacionales de las primeras es posible inferir que estas diferencias se explican por el menor nivel económico, antes que por un menor aprecio por la lectura. 
34 STUdiantes MigRANTES EN ESCUELAS PÚBLICAS CHILENAS - D. Castillo, E. SantaCruz y A. Vega

Tabla 6

Cuántos libros hay en el hogar del estudiante

\begin{tabular}{lrrr}
\hline \multirow{2}{*}{ Cuántos libros hay en el hogar } & \multicolumn{2}{c}{ ¿Es usted extranjero? } & \multirow{2}{*}{ Total } \\
\cline { 2 - 3 } & \multicolumn{1}{c}{ Sín } & No & \\
\hline Ninguno & 8,9 & 2,6 & 4,7 \\
Menos de 10 & 53,7 & 24,9 & 34,3 \\
Entre 10 y 50 & 34,1 & 44,5 & 41,1 \\
Entre 51 y 100 & 3,3 & 20,4 & 14,8 \\
Entre 101 y 500 & - & 6,1 & 4,1 \\
Más de 500 & & 1,6 & 1,1 \\
Total & 100,0 & 100,0 & 100,0 \\
\hline
\end{tabular}

Fuente: Elaboración propia.

$\mathrm{Al}$ ser consultados respecto de la cantidad de establecimientos educacionales a los cuales ha asistido el o la estudiante en su itinerario educativo, ambos grupos declararon mayoritariamente que estos han asistido a un solo establecimiento. Cabe destacar, en términos de itinerancia entre un establecimiento y otro, que los apoderados nacionales declaran, en mayor proporción que los migrantes, que sus pupilos han asistido a tres o más establecimientos distintos en su historia educativa.

Tabla 7

¿A cuántos establecimientos educacionales distintos ha asistido el estudiante desde $1^{\circ}$ básico?

\begin{tabular}{lrrrr}
\hline \multirow{2}{*}{$\begin{array}{l}\text { ¿A cuántos establecimientos educacionales distintos } \\
\text { ha asistido el estudiante desde } 1^{\circ} \text { básico? }\end{array}$} & \multicolumn{2}{c}{ ¿Es usted } \\
\cline { 2 - 3 } & \multicolumn{2}{c}{ extranjero? } & \multirow{2}{*}{ Total } \\
Siempre ha estado en el mismo establecimiento. & 58,1 & 52,4 & 54,2 \\
Solo un establecimiento anteriormente. & 17,4 & 18,3 & 18,0 \\
Dos establecimientos distintos. & 16,9 & 13,7 & 14,8 \\
Tres o más establecimientos distintos. & 7,6 & 15,5 & 13,0 \\
Total & 100,0 & 100,0 & 100,0 \\
\hline
\end{tabular}

Fuente: Elaboración propia.

Las siguientes tablas dan cuenta de las trayectorias educativas y las expectativas puestas en el futuro educativo. En este contexto, en primer término, si bien ambas poblaciones afirman mayoritariamente que sus pupilos nunca han repetido de curso, la población migrante es la que declara una mejor trayectoria. Así, según lo expresado por estas poblaciones, los estudiantes migrantes tendrían experiencias educativas más exitosas que sus pares locales en términos de repitencia. 
Tabla 8

¿Cuántas veces el estudiante ha repetido de curso?

\begin{tabular}{lrrr}
\hline \multirow{2}{*}{ Cuántas veces el estudiante ha repetido de curso? } & \multicolumn{2}{c}{ ¿Es usted extranjero? } & \multirow{2}{*}{ Total } \\
\cline { 2 - 3 } & \multicolumn{1}{c}{ Sí } & No & \\
\hline Nunca ha repetido & 90,0 & 84,5 & 86,3 \\
Una vez & 10,0 & 11,8 & 11,2 \\
Dos veces & 0,0 & 3,0 & 2,0 \\
Tres o más veces & 0,0 & 0,6 & 0,4 \\
Total & 100,0 & 100,0 & 100,0 \\
\hline
\end{tabular}

Fuente: Elaboración propia.

En cuanto a las expectativas educativas que los apoderados declaran para sus pupilos, no existen diferencias estadísticamente significativas entre ambos colectivos. Es así como, sobre un 85\% de ambas poblaciones declaran tener expectativas de estudios a nivel superior para sus pupilos. En este sentido, es interesante observar que ambos tipos de apoderados tienen expectativas de desarrollo vinculadas con la formación académica y profesional, lo que podría ser una herramienta para acceder a mejores fuentes laborales.

Tabla 9

¿Cuál cree usted que es el nivel de educación más alto que el estudiante completará a futuro?

\begin{tabular}{|c|c|c|c|}
\hline \multirow{2}{*}{$\begin{array}{l}\text { ¿Cuál cree usted que es el nivel de educación más ato } \\
\text { que el estudiante completará a futuro? }\end{array}$} & \multicolumn{2}{|c|}{$\begin{array}{l}\text { ¿Es usted } \\
\text { extranjero? }\end{array}$} & \multirow[t]{2}{*}{ Total } \\
\hline & Sí & No & \\
\hline No creo que complete $4^{\circ}$ año de enseñanza media & 2,1 & 0,2 & 0,8 \\
\hline $4^{\circ}$ año de enseñanza media técnico-profesional & 8,4 & 10,7 & 9,9 \\
\hline $4^{\circ}$ año de enseñanza media científico-humanista & 0,4 & 1,2 & 1,0 \\
\hline $\begin{array}{l}\text { Una carrera en un instituto profesional o centro de } \\
\text { formación técnica }\end{array}$ & 21,4 & 20,9 & 21,1 \\
\hline Una carrera en la universidad & 52,5 & 51,0 & 51,5 \\
\hline Estudios de posgrado & 15,1 & 16,0 & 15,7 \\
\hline Total & 100,0 & 100,0 & 100,0 \\
\hline
\end{tabular}

Fuente: Elaboración propia.

Por otra parte, se consultó a los apoderados cuál era su percepción respecto del trato que se da a los extranjeros en Chile. Llama la atención, en virtud de las innumerables ocasiones en las cuales los medios de comunicación informan acerca del fenómeno 
de la discriminación en Chile, que los apoderados migrantes declaren percibir el trato que los chilenos les brindan de forma más positiva que sus pares nativos. Frente a estas percepciones, sería interesante conocer cuáles serían los factores que subyacen en ellas ya que, entre otras hipótesis, podrían existir a la base aspectos culturales, de expectativas o costumbres.

Tabla 10

¿Cómo diría usted que los chilenos tratan a los extranjeros que residen en Chile?

\begin{tabular}{lrrr}
\hline Trato a las personas extranjeras que residen en Chile iEs usted extranjero? & \multirow{2}{*}{ Total } \\
\cline { 2 - 3 } & \multicolumn{1}{c}{ Sín } & \multicolumn{1}{c}{ No } & \\
\hline Con desprecio & 4,1 & 7,1 & 6,1 \\
Con desconfianza & 11,2 & 25,1 & 20,5 \\
Con indiferencia & 18,6 & 14,3 & 15,7 \\
Con normalidad & 50,0 & 44,0 & 46,0 \\
Con amabilidad & 16,1 & 9,6 & 11,7 \\
Total & 100,0 & 100,0 & 100,0 \\
\hline
\end{tabular}

Fuente: Elaboración propia.

Ahora, en cuanto a la percepción de discriminación ejercida tanto hacia apoderados migrantes como locales, la siguiente tabla muestra que los apoderados nativos se han percibido menos discriminados que sus pares migrantes. Los datos muestran una diferencia significativa entre ambos, expresándose en que casi el $60 \%$ de los apoderados migrantes señalan haber vivido experiencias de discriminación en Chile, lo que contrasta con el 35\% de los nacionales que dice haber experimentado situaciones similares.

Tabla 11

Frecuencia de discriminación percibida por el apoderado

\begin{tabular}{lccc}
\hline \multirow{2}{*}{ Frecuencia } & \multicolumn{2}{c}{ ¿Es usted extranjero? } & \multirow{2}{*}{ Total } \\
\cline { 2 - 3 } & Sín & No & \\
\hline Muchas veces & 3,2 & 0,6 & 1,5 \\
Algunas veces & 20,3 & 7,1 & 11,6 \\
Pocas veces & 35,1 & 28,8 & 31,0 \\
Nunca & 41,4 & 63,5 & 55,9 \\
Total & $100,0 \%$ & $100,0 \%$ & $100,0 \%$ \\
\hline
\end{tabular}

Fuente: Elaboración propia. 


\subsection{Análisis sociométrico sobre las redes de amistad}

Las respuestas reportadas por los estudiantes permiten la construcción de tipos sociométricos, cuya distribución no muestra, en términos generales, diferencias estadísticamente significativas entre colectivos en términos agregados. Como aparece en la Tabla 12, los migrantes aparecen menos en la categoría de "Preferidos", pero también poseen menor porcentaje en la de "Controvertidos". En su caso, las categorías "Ignorados" y "Medios", que hablan de aislamiento o menor amplitud social en las elecciones y/o rechazos respectivamente, tienen un mayor peso. Por el contrario, los nacionales muestran mayor intensidad en las preferencias y/o rechazos de lo que se observa para el grupo de los migrantes, lo que se expresa en un mayor porcentaje en las categorías de "Preferidos", "Rechazados" y "Controvertidos".

Tabla 12

Tipos sociométricos a partir de las relaciones de amistad/rechazo

\begin{tabular}{lccr}
\hline Categoría & Nacional & Migrante & Total \\
\hline Preferidos & 25,4 & 22,8 & 24,5 \\
Rechazados & 7,9 & 7,2 & 7,7 \\
Ignorados & 12,4 & 17,4 & 14,0 \\
Controvertidos & 14,1 & 9,0 & 12,5 \\
Medios & 40,3 & 43,7 & 41,4 \\
Total & 100,0 & 100,0 & 100,0 \\
\hline
\end{tabular}

Fuente: Elaboración propia.

En las escuelas con baja concentración de migrantes, el peso de la categoría de "Ignorados" en los estudiantes migrantes es notoriamente mayor que la que se observa en escuelas con alta concentración de migrantes, triplicando la proporción de nacionales en la misma categoría. Dicho de otra manera, cuando en los establecimientos asiste una mayor cantidad de migrantes, estos tienden a ser menos ignorados, aunque este cambio se realiza en beneficio del crecimiento de las categorías de "Controvertidos" y "Rechazados". Esto es muy diferente a lo observado cuando se les consultó por los estudiantes con quiénes jugaban (o no), pues respecto de aquello la distancia entre nacionales y migrantes en la categoría de "Ignorados" disminuía ostensiblemente. Esto se puede resumir en que los migrantes son más escogidos, pero también 
38 STUdiAnTES MigRANTES EN ESCUELAS PÚBLICAS CHILENAS - D. Castillo, E. SantaCruz y A. Vega

más rechazados, al momento de decidir con quién jugar, y menos considerados cuando se trata de seleccionarlos (o no) como amigos.

Tabla 13

Tipos sociométricos a partir de las relaciones de amistad/rechazo escuela con alta y medio-baja concentración

\begin{tabular}{lcccccc}
\hline \multirow{2}{*}{ Categoría } & \multicolumn{3}{c}{ Alta-media concentración } & \multicolumn{3}{c}{ Media-baja concentración } \\
\cline { 2 - 6 } & Nacional & Migrante & Total & Nacional & Migrante & Total \\
\hline Preferidos & 21,8 & 22,5 & 22,1 & 28,1 & 23,7 & 27,4 \\
Rechazados & 7,7 & 9,3 & 8,4 & 8,0 & & 6,8 \\
Ignorados & 16,7 & 13,2 & 15,1 & 9,0 & 31,6 & 12,7 \\
Controvertidos & 14,1 & 11,6 & 13,0 & 14,1 & & 11,8 \\
Medios & 39,7 & 43,4 & 41,4 & 40,7 & 44,7 & 41,4 \\
Total & 100,0 & 100,0 & 100,0 & 100,0 & 100,0 & 100,0 \\
\hline
\end{tabular}

Fuente: Elaboración propia.

En función de lo anterior, a continuación se grafican las redes de relaciones en la dimensión amistad o las redes de amistades, organizadas en función de las declaraciones de los estudiantes que nominan a sus amistades por curso. La representación es expresiva de la forma en que se vinculan los estudiantes en términos de redes de amistad en una escuela con media-baja concentración de población migrante. Según la información proporcionada por los estudiantes, el curso está constituido por, al menos, cuatro subgrupos de amistades, en los cuales es posible apreciar que la inclusión de estudiantes migrantes en redes de amistad adquiere diferente estructura. Por un lado, se encuentra un triángulo, donde tres estudiantes de origen haitiano se eligen recíprocamente, y solo uno de ellos escoge a un estudiante nacional como su amigo. Por el contrario, no reciben ninguna mención por parte de sus compañeros de clase. Esta experiencia de aislamiento al interior del curso no corresponde, sin embargo, a la que manifiestan los restantes estudiantes migrantes de la sala. En el caso del estudiante 17, de nacionalidad peruana, este se encuentra completamente integrado a una gran cadena de elecciones con estudiantes de origen chileno. Incluso, con dos de ellos, establece elecciones recíprocas, lo que expresaría una intensidad significativa en el sentimiento manifestado. Por último, las estudiantes 16 y 22, de origen migrante, logran establecer relaciones de amistad con estudiantes no migrantes, 
aunque estas últimas no siempre las escogen como sus mejores amigas. En suma, la red de relaciones que se grafica en esta escuela de baja-mediana concentración de migrantes muestra la existencia de experiencias disímiles entre sí, la que van desde experiencias donde la condición nacional no parece tener ninguna relevancia a otras donde el género y el origen nacional parece tener una fuerte incidencia.

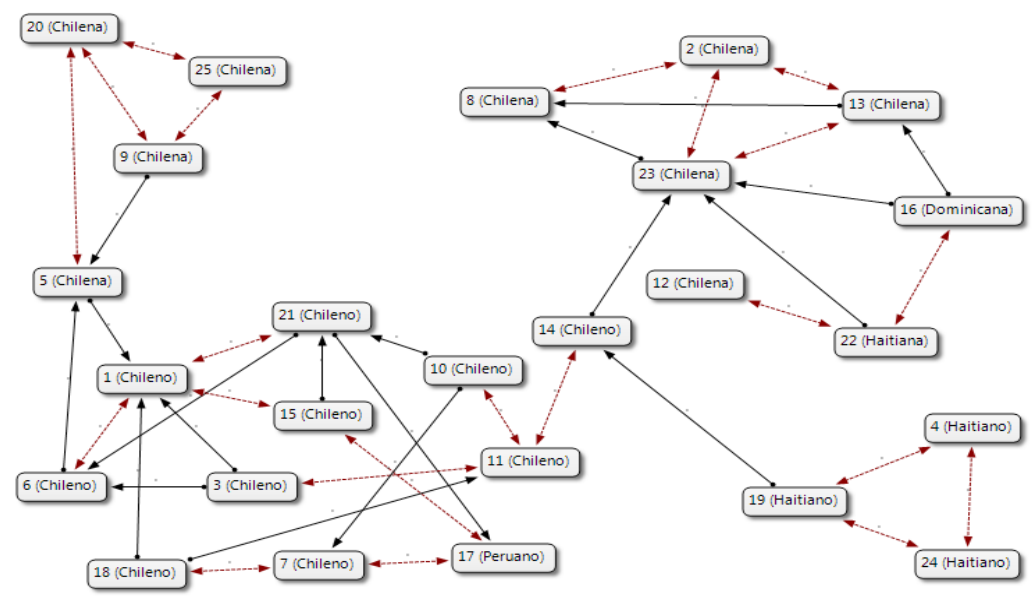

Figura 1. Redes de amistades en un curso de escuela de medio-baja concentración migrante.

Fuente: Elaboración propia.

Por otra parte, en la Figura 2, que corresponde a un curso con una alta concentración de migrantes, los resultados tienden a asemejarse a la estructura de relaciones de la figura anterior, pero en este caso se pueden identificar cinco subgrupos, relativamente cerrados entre sí, con fuertes relaciones recíprocas y vínculos con los otros subgrupos dependientes de un solo estudiante de cada cadena. Ese rol lo ocupan estudiantes chilenos y migrantes, identificándose al 36, 32 y 33 en el primer subgrupo, y al 35 y 5 en el segundo. Dos de los subgrupos tienen una composición mixta en términos de condición nacional, mientras que los otros tres agrupan a estudiantes de similar origen. Así, se puede encontrar un subgrupo compuesto exclusivamente por chilenas (17, 25, 3, 36, 6, 29, 23 y 22); mientras que los otros dos subgrupos están conformados casi exclusivamente 
por estudiantes migrantes, uno de mujeres y otro de hombres. Esta característica de separación de las redes de amistad por género está fuertemente presente en los cursos analizados, por lo que la condición nacional de los estudiantes antes que desafiar dicho principio de estructuración de las redes de amistad parece haber convivido y dialogado con él.



Figura 2. Redes de amistad en un curso de escuela con alta concentración migrante.

Fuente: Elaboración propia.

Como se aprecia en los ejemplos anteriores, existen experiencias distintas en las relaciones de amistad que se establecen entre los estudiantes en escuelas con distinto grado de concentración de estudiantes migrantes. Un rasgo que se tiende a presentar es la fuerte dependencia de las redes sociales de amistad con el género de los estudiantes, siendo un elemento relevante para la conformación de los subgrupos. Así mismo, la condición nacional aparece vinculada con esta situación, siendo más frecuente la estructuración de subgrupos de migrantes cuando esta condición concurre con el hecho de que sean estudiantes mujeres. Una excepción a esto, se observó en el caso de los estudiantes de origen haitiano, quienes en el marco de esta investigación no aparecían integrados de modo intenso a redes de amistad con nacionales o migrantes de otras nacionalidades. 


\subsection{Análisis sociométrico sobre el trabajo escolar}

El trabajo escolar es una actividad que se realiza mayoritariamente al interior del aula y que se encuentra fuertemente regulada por el docente. Son los profesores los que determinan cuándo se realiza este y qué características asume la actividad en concreto. Pese a esto, en estas actividades son los propios estudiantes quienes deciden con quién trabajar.

Tabla 14

Tipos sociométricos en la variable de trabajo escolar

\begin{tabular}{lccc}
\hline Categoría & Nacional & Migrante & Total \\
\hline Preferidos & 14,4 & 14,4 & 14,4 \\
Rechazados & 7,9 & 4,2 & 6,7 \\
Ignorados & 7,9 & 15,0 & 10,2 \\
Controvertidos & 23,1 & 19,2 & 21,8 \\
Medios & 46,8 & 47,3 & 46,9 \\
Total & 100,0 & 100,0 & 100,0 \\
\hline
\end{tabular}

Fuente: Elaboración propia.

Como se evidencia en la Tabla 14 , la proporción de migrantes que es encasillado en la categoría de "Rechazados" es significativamente menor a lo que registra el subgrupo de nacionales. $\mathrm{Al}$ mismo tiempo, los migrantes reafirman la tendencia observada en la dimensión anterior de mostrar una mayor presencia en la categoría de "Ignorados". Este menor rechazo hacia los migrantes al momento de establecer relaciones para hacer trabajos escolares está más presente en el caso de las escuelas con una concentración media-baja de migrantes. Este dato, en principio indicador de inclusión, debe ser tomado con cautela, dado que esta menor reticencia a trabajar con migrantes se ve acompañada de un significativo incremento de los "Ignorados" en los colegios con baja presencia de migrantes. 
42 STUdiantes MigRANTES EN ESCUELAS PÚBLICAS CHILENAS - D. Castillo, E. SantaCruz y A. Vega

Tabla 15

Tipos sociométricos en la variable de trabajo escolar

\begin{tabular}{lcccc}
\hline \multirow{2}{*}{ Categoría } & \multicolumn{2}{c}{ Alta-media concentración } & \multicolumn{2}{c}{ Medio-baja concentración } \\
\cline { 2 - 5 } & Nacional & Migrante & Nacional & Migrante \\
\hline Preferidos & 8,3 & 14,0 & 19,1 & 15,8 \\
Rechazados & 7,7 & 4,7 & 8,0 & 2,6 \\
Ignorados & 9,9 & 14,0 & 7,0 & 18,4 \\
Controvertidos & 23,1 & 18,6 & 23,1 & 21,1 \\
Medios & 51,9 & 48,8 & 42,7 & 42,1 \\
Total & 100,0 & 100,0 & 100,0 & 100,0 \\
\hline
\end{tabular}

Fuente: Elaboración propia.

La menor tendencia a ser rechazado para trabajar dentro del aula que se advierte en el caso de los migrantes no es extensible a todos los colectivos nacionales en los establecimientos analizados. Del mismo modo, cabe señalar que en esta dimensión los tipos sociométricos se distribuyen de un modo significativamente distinto entre hombres y mujeres. En este sentido, las diferencias entre colectivos migrantes y nacionales no parecen ser tan marcadas cuando la comparación se establece entre hombres; mientras tanto, en el caso de las mujeres, las diferencias son aún mayores.

La representación gráfica de las relaciones entre estudiantes en el ámbito del trabajo escolar muestra ciertas similitudes con lo observado en el caso de las redes de amistad. En la Figura 3, por ejemplo, se advierten cuatro subgrupos de estudiantes marcadamente diferenciados. El primero de ellos, está conformado exclusivamente por hombres, integrado principalmente por chilenos, y sus relaciones de amistad con los otros dos subgrupos son inexistentes o dependen de un sujeto (alumno 5) que cumple las veces de puente entre dos colectivos. El siguiente subgrupo, conformado en su centralidad por mujeres nacionales (2, 8, 23 y 13), cuenta en sus márgenes con la pertenencia de dos mujeres migrantes, quienes manifiestan tener relaciones de amistad con ellas, aun cuando esto no parece ser recíproco. Un tercer subgrupo corresponde a una pareja de estudiantes nacionales, las que tienen una fuerte relación entre sí, pero se encuentran relativamente desconectadas de los demás subgrupos. Por último, se observa el caso del subgrupo de estudiantes de origen haitiano, quienes al momento de escoger o ser elegidos para 
trabajar en clases no hacen ni reciben menciones de ningún individuo por fuera del subgrupo. Corresponde a una estructura de camarilla, con una fuerte cohesión interna, pero una nula relación con los demás subgrupos.

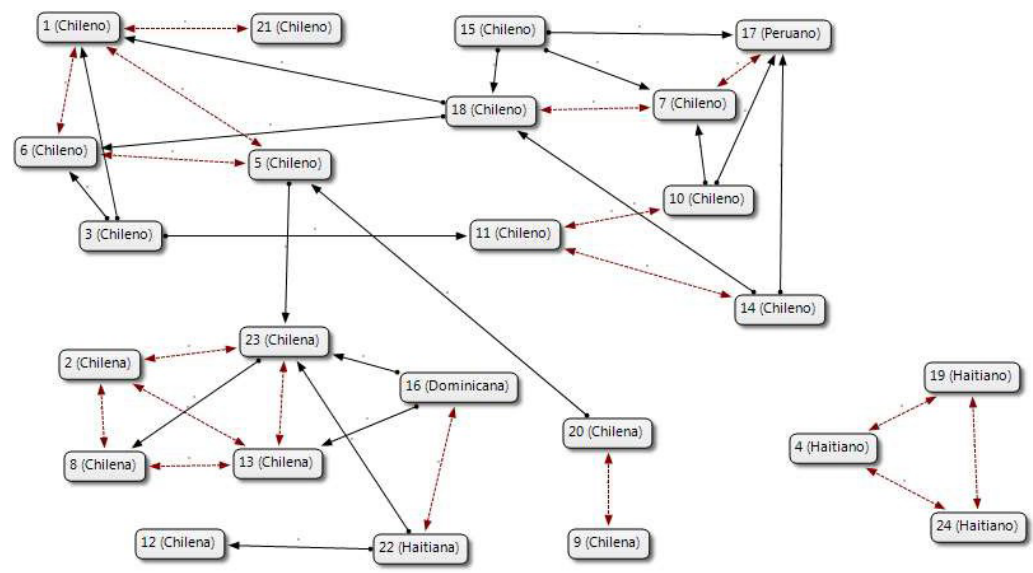

Figura 3. Redes de trabajo laboral curso de escuela de baja concentración migrante.

Fuente: Elaboración propia.

El panorama anterior se ve modificado en la Figura 4, donde el género es un elemento diferenciador al describir las características de los distintos subgrupos que se establecen en el aula de esa escuela con alta presencia de migrantes, sean de primera como de segunda generación. En este curso se identifican cuatro subgrupos, con tamaños, composición y estructuras diferentes entre sí. 


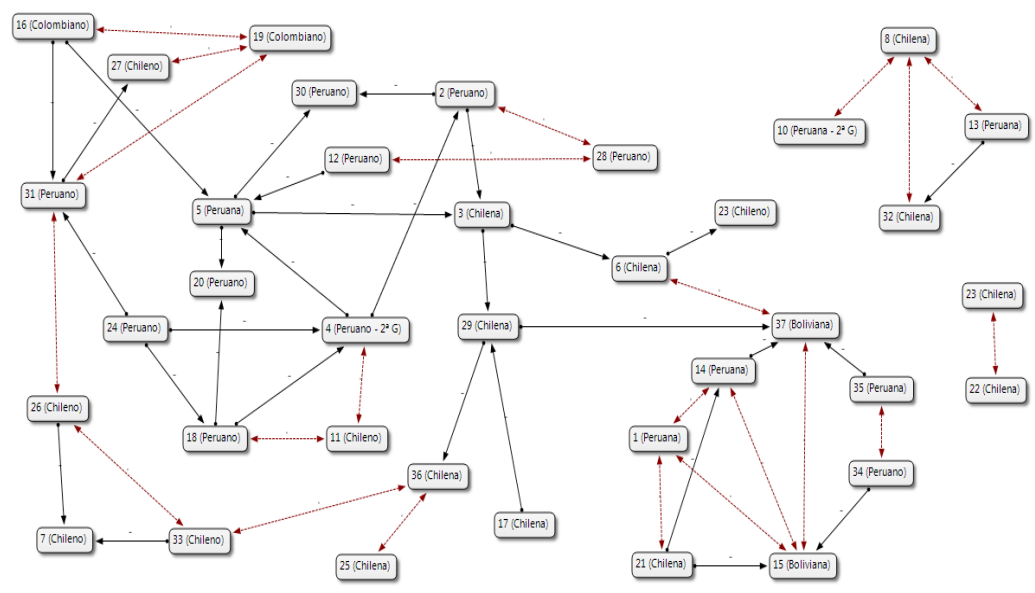

Figura 4. Redes de trabajo laboral en un curso de escuela con alta concentración migrante. Fuente: Elaboración propia.

En primer lugar, se encuentra una red donde se relaciona la gran parte de los estudiantes, mayoritariamente hombres y con una fuerte integración de los alumnos en virtud de su origen nacional. No se advierten posiciones centrales en la red y se establecen relaciones recíprocas de elección entre chilenos y migrantes. Un segundo subgrupo corresponde a una pareja de estudiantes de origen nacional, quienes tienen una nula relación con individuos de otras redes al momento de hacer deberes escolares. Un tercer subgrupo corresponde a cuatro mujeres, dos chilenas y dos de origen migrante, quienes establecen fuertes relaciones entre sí y aparecen aisladas del resto en este ámbito. Se puede identificar un cuarto subgrupo de mayor cantidad de componentes — un total de nueve estudiantes-, quienes son, en su mayoría, migrantes y mujeres. En suma, en este curso predomina el género como criterio estructurador de las relaciones para trabajar en el aula, observándose que la condición nacional se subordina y parece tener una importancia secundaria.

\section{Conclusiones}

La creciente llegada de migrantes al sistema escolar chileno ha develado la carencia de políticas específicas orientadas a su incorporación adecuada en las instituciones escolares. La tardía generación 
de conocimientos respecto de las expectativas, características y necesidades de un colectivo heterogéneo, además de la debilidad de las estructuras institucionales destinadas a favorecer su inclusión, ha generado una desigual integración de estudiantes y apoderados migrantes al sistema escolar, dimensión clave en los modos de incorporación de estas familias a la sociedad de recepción. Este artículo ha buscado ofrecer mayor luz en torno a las características de esta población, pero también iniciar el conocimiento de las dinámicas de inclusión al interior de espacios cotidianos del mundo escolar, pero sumamente relevantes para la inserción de los estudiantes.

La investigación dio cuenta de similitudes y diferencias en el caso de las familias migrantes y no migrantes que es preciso mencionar. Pese a contar con un capital cultural objetivado similar a los no migrantes, en las familias migrantes se advierte un marcado proceso de inserción en condiciones de subordinación al mercado laboral, estando la mitad de ellas ocupadas en trabajos no calificados y/o en trabajos de servicio o en el comercio, porcentaje que duplica a la proporción de chilenos. Los apoderados de familias migrantes también cuentan con menos ingresos, arriendan y no son dueños del lugar donde viven, y sus condiciones de habitabilidad son más precarias; así mismo, cuentan con menos recursos culturales en sus hogares (libros, internet y computadores). En términos de su percepción acerca de la discriminación, las familias migrantes sostienen una mirada dual: por un lado, en términos absolutos, en una menor proporción que los no migrantes señalan que la sociedad chilena es discriminatoria; mientras que, por otra parte, una proporción no menor sostiene que han vivido personalmente situaciones de discriminación.

Respecto de las relaciones sociales establecidas en el ámbito del juego y del trabajo, los esquemas muestran que, en general, la inclusión los estudiantes migrantes en la sala de clases es aún insuficiente, pese a que varía dependiendo de los contextos específicos. Es así como, en la medida en que aumenta la concentración de comunidades migrantes, tiende a crecer la distancia social entre los colectivos. Por eso es más fuerte donde el resultado de la disputa aún no está resuelto — en las escuelas con poblaciones migrantes y no migrantes más equilibradas 
hay mayor tensión-, y se produce mayor tensión con los colectivos de ciertos países más que con otros (colombianos y haitianos, que con peruanos y bolivianos). En el caso del trabajo escolar, pero en particular en las redes de amistad, los principios de estructuración de la interacción en el espacio escolar están fuertemente mediados por el género y la condición nacional. Sin embargo, esto no opera de similar modo para todos los colectivos nacionales. En el caso de los estudiantes haitianos se aprecia un significativo aislamiento en relación, por ejemplo, con lo que se aprecia en el caso de estudiantes de origen peruano, quienes parecen interactuar y ser parte de redes con estudiantes de origen chileno en mayor porcentaje. Con todo, lo que muestra en mayor medida el test sociométrico es que los estudiantes migrantes poseen mayor probabilidad de estar en la categoría de los Ignorados.

Este escenario empírico tiende a corroborar lo que señala parte de la evidencia comparada revisada, donde se indica que la convivencia entre colectivos distintos no soluciona por sí misma las dificultades de integración que se generan. Es por esta razón que, aunque las escuelas chilenas actúan en el marco general de un sistema educativo de mercado, la política general y escolar debiera intervenir en la distribución de los estudiantes, para atender los efectos de segregación que se están produciendo en muchos establecimientos. Todo lo anterior requiere que la política pública en educación ingrese activamente y regule la construcción de "guetos", pero también a través de una intervención a nivel de micropolíticas en los espacios escolares.

\section{Referencias}

Abad, L.V. (1993). La educación intercultural como propuesta de integración. En L.V. Abad, A. Cucó, \& A. Izquierdo (Eds.), Inmigración, pluralismo y tolerancia (pp. 9-69). Madrid: Ed. Popular.

Abrantes, P. (2008). Individualización y exclusión. La transición a la secundaria en el centro de Madrid. Revista de la Asociación de Sociología de la Educación, 1(2), 5-21.

Alegre, M.A., Benito, R. \& González, S. (2006). Immigrants als instituts: L'acollida vista pels seus protagonistes. Barcelona: Fundación Jaume Boffil. 
Bernstein, B. (1994). La estructura del discurso pedagógico. Madrid: Morata.

Bezanilla, J.M. (2011). Sociometría. Un método de investigación social. México D.F.: PEI Editorial.

Borjas, G. (1995). The economic benefits from immigration. Journal of Economic Perspectives, 9(2), 3-22. https://doi.org/10.1257/jep.9.2.3

Burgess, S., Wilson, D., \& Lupton, R. (2005). Parallel lives? Ethnic segregation in schools and neighbourhoods. Urban Studies, 42(7), 1027-1056. https://doi.org/10.1080/00420980500120741

Calero, J. y Waisgrais, S. (febrero, 2009). Rendimientos educativos de los alumnos inmigrantes: identificación de la incidencia de la condición de inmigrante y de los peer effects. Trabajo presentado en el XVI Encuentro de Economía Pública, Universidad de Granada, Granada, España.

Carbonell, F. (1999). Desigualdad social, diversidad cultural y educación. En E. Aja, F. Carbonell, Colectivo Ioé, J. Funes, \& I. Vila (Coord.), La inmigración extranjera en España. Los retos educativos (pp. 99-118). Barcelona: Fundación La Caixa.

Cárdenas, M. (2006). Y verás cómo quieren en Chile...: Un estudio sobre el prejuicio hacia los inmigrantes bolivianos por parte de los jóvenes chilenos. Última Década, 14(24), 99-124. https://doi.org/10.4067/s0718-22362006000100006

Castillo, D. y Gajardo, C. (enero, 2016). Inclusión y procesos de escolarización en menores migrantes que asisten a establecimientos de educación básica. Ponencia para el IX Congreso Internacional - XV Congreso Nacional de Investigadores en Educación, INVEDUC. Universidad de Los Lagos, Osorno, Chile.

Castles, S. \& Davidson, A. (2000). Citizenship and migration: globalization and the politics of belonging. New York: Routledge.

Colectivo Ioé (1999a). Inmigrantes, trabajadores, ciudadanos. Una visión de las migraciones desde España. Valencia: Universidad de Valencia.

Colectivo Ioé (1999b). El desafío intercultural españoles ante la inmigración. En E. Aja, F. Carbonell, Colectivo Ioé, J. Funes e I. Vila (Coord.), La inmigración extranjera en España. Los retos educativos (pp. 13-68). Barcelona: Fundación La Caixa.

Colectivo Ioé (2002). Inmigración, escuela y mercado de trabajo. Una radiografía actualizada. Barcelona: Fundación La Caixa.

Corporación Opción. (2014). Niñez y adolescencia en Chile: las cifras. Datos estadísticos y principales estudios. Santiago de Chile: LOM. 
48 STUdiantes MigRANTES EN ESCUELAS PÚBLICAS CHILENAS - D. Castillo, E. SantaCruz y A. Vega

Frankenberg, E., Lee, C., \& Orfield, G. (2003) A multiracial society with segregated schools: are we losing the dream? Civil Rights Project, Cambridge, MA: Harvard University.

Gallimore, R. \& Goldenberg, C. (2001). Analyzing cultural models and settings to connect minority achievement and school improvement research. Educational Psychologist, 36(1), 45-56. https://doi.org/10.1207/s15326985ep3601_5

García-Bacete, FJ., I. Sureda, I., y Monjas, M.I. (2010). El rechazo entre iguales en la educación primaria: una panorámica general. Anales de Psicología, 26, 123-136.

García, J. y Granados, A. (2002). Inmigración, educación e interculturalidad, Migrance, 21, 182-198.

Giménez, C. y Malgesini, G. (2000). Guía de conceptos sobre migraciones, racismo e interculturalidad. Madrid: Catarata.

Instituto Nacional de Estadísticas, INE. (2003). Sintesis de resultados. Censo 2002. Santiago de Chile: Autor.

Instituto Nacional de Estadísticas, INE. (2013). Resultados XVIII Censo de población 2012. Santiago de Chile: Autor.

Navas, L. y Sánchez, A. (2010). Actitudes de los estudiantes de Pedagogía de las regiones del Bío Bío y la Araucanía de Chile hacia la presencia de niños inmigrantes en la escuela: análisis diferenciales. Psykhe, 19(1), 47-60. https://doi.org/10.4067/s0718-22282010000100004

Organización para la Cooperación y el Desarrollo Económicos, OCDE (2011). ¿Cómo se están adaptando los sistemas escolares al creciente número de estudiantes inmigrantes?, Pisa in Focus. Paris: Autor.

Pávez, I. (2012). Inmigración y racismo: experiencias de niñez peruana en Santiago de Chile. Si Somos Americanos, 12(1), 75-99. https://doi.org/10.4067/s0719-09482012000100004

Pettigrew, T.F. \& Meertens, R.W. (1995). Subtle and blatant prejudice in western Europe. European Journal of Social Psychology, 25(1), 57-75. https://doi.org/10.1002/ejsp.2420250106

Portes, A. (1995). Economic sociology of immigration: a conceptual overview. En A. Portes (Ed.), The economic sociology of immigration: essays on network, ethnicity and entrepreneurship (pp. 1-41). New York: Russell Sage Foundation.

Portes, A. \& Rumbaut, R. (1990). Immigrant America: a portrait. Los Angeles: University of California Press. 
Portes, A. \& Zhou, M. (1993). "The new second generation: segmented assimilation and its variants" en Annals of the American Academy of Political and Social Science, 530(1), 74-96.

Rivkin, S. (2000). School desegregation, academic attainment, and earnings. Journal of Human Resources, 35, 333-346. https://doi.org/10.2307/146328

Schiappacasse, P. (2008). Segregación residencial y nichos étnicos de los inmigrantes internacionales en el Área Metropolitana de Santiago. Revista Geografía Norte Grande, 39, 21-38.

Stark, A. (1993). What's the matter with business ethics. Recuperado de https:// hbr.org/1993/05/whats-the-matter-with-business-ethics.

Stefoni, C. y Fernández, R. (2011). Mujeres inmigrantes en el trabajo doméstico: entre el servilismo y los derechos. En C. Stefoni (Ed.): Mujeres inmigrantes en Chile, ¿mano de obra o trabajadoras con derechos? (pp. 43-72). Santiago de Chile: Ediciones Universidad Alberto Hurtado.

Thayer, L. E. (2007). Inmigrantes ecuatorianos en la Comunidad de Madrid: la apropiación del espacio y la expropiación del tiempo. Madrid: Complutense.

Thayer, L.E (2013). Expectativas de reconocimiento y estrategias de incorporación: la construcción de trayectorias degradadas en migrantes latinoamericanos residentes en la Región Metropolitana de Santiago. Polis, 12(35), 1-18.

https://doi.org/10.4067/s0718-65682013000200012

Tijoux, M. E. (2013). Las escuelas de la inmigración en la ciudad de Santiago: Elementos para una educación contra el racismo. Polis, 12(35), 287 307. https://doi.org/10.4067/s0718-65682013000200013

Recibido: 25/04/2018

Aceptado: 16/10/2018 